\title{
Organization of silicon nanocrystals by localized electrochemical etching
}

Asma Ayari-Kanoun, Dominique Drouin, Jacques Beauvais, Vladimir Lysenko, Tetyana Nychyporuk, and Abdelkader Souifi

Citation: Appl. Phys. Lett. 95, 153105 (2009); doi: 10.1063/1.3247884

View online: http://dx.doi.org/10.1063/1.3247884

View Table of Contents: http://aip.scitation.org/toc/apl/95/15

Published by the American Institute of Physics

Save your money for your research.

It's now FREE to publish with us -

no page, color or publication charges apply. 


\title{
Organization of silicon nanocrystals by localized electrochemical etching
}

\author{
Asma Ayari-Kanoun, ${ }^{1, a)}$ Dominique Drouin, ${ }^{1}$ Jacques Beauvais, ${ }^{1}$ Vladimir Lysenko, ${ }^{2}$ \\ Tetyana Nychyporuk, ${ }^{2}$ and Abdelkader Souifi ${ }^{2}$ \\ ${ }^{1}$ Department of Electrical Engineering, Université de Sherbrooke, Sherbrooke, Québec JIK 2RI, Canada \\ ${ }^{2}$ Institut des Nanotechnologies de Lyon (INL), UMR-CNRS 5270, INSA de Lyon, 7 Avenue Jean \\ Capelle, 69621 Villeurbanne Cedex, France
}

(Received 6 July 2009; accepted 23 September 2009; published online 13 October 2009)

\begin{abstract}
An approach to form a monolayer of organized silicon nanocrystals on a monocrystalline Si wafer is reported. Ordered arrays of nanoholes in a silicon nitride layer were obtained by combining electron beam lithography and plasma etching. Then, a short electrochemical etching current pulse led to formation of a single Si nanocrystal per each nanohole. As a result, high quality silicon nanocrystal arrays were formed with well controlled and reproducible morphologies. In future, this approach can be used to fabricate single electron devices. (c) 2009 American Institute of Physics. [doi:10.1063/1.3247884]
\end{abstract}

In recent years, the organization of silicon nanocrystals became one of the most important concerns for large scale integration of nanoelectronic devices, such as single electron transistors ${ }^{1}$ and nonvolatile memories. ${ }^{2}$ Several techniques were proposed for nanocrystal organization. In particular, self-organization processes have recently attracted increasing attention by using prestructured surfaces as templates to organize nanocrystals grown naturally or artificially. The one kind of approaches concerns the natural prestructuring of the surface resulted from the creation of a periodic strain network defining a regular array of nucleation and growth sites. ${ }^{3}$ This method exhibits the advantage of being quick and is applicable on all types of surfaces with sizes up to a few $\mathrm{mm}^{2}$. However, using this approach, rectilinear strain networks are difficult to achieve and the obtained structures are usually inhomogeneous in size. ${ }^{4,5}$ Second kind of approaches concerns artificial prestructuring, which consists of creating a periodic pattern with high-resolution lithography combined with etching techniques ${ }^{6,7}$ or using porous anodic alumina as template. ${ }^{8}$ This kind of approaches is much more precise and achieves a good resolution. It is the latter that was used during the present work to create a patterned nanohole array in a silicon nitride thin film. These templates will define the localization of silicon nanocrystals realized by electrochemical etching of the substrate.

It was recently shown that using short single pulses of current in a low concentration of hydrofluoric acid electrolytes and a current density in the transition regime between pore formation and electropolishing, allows the formation of a monolayer of laterally separated silicon nanocrystals at the surface of silicon wafers. ${ }^{9,10}$ Depending on substrates and anodization parameters, high quality silicon nanocrystal arrays can be formed with well controlled and reproducible morphologies. These nanocrystals have the same doping level and crystallographic orientation as the initial substrate. Structure, chemical composition, and light emitting properties of the Si nanocrystals obtained by this method were recently studied in details. ${ }^{11,12}$ However, no attempt has been reported regarding the means of localizing these nanocrys-

\footnotetext{
${ }^{\text {a) }}$ Author to whom correspondence should be addressed. Electronic mail: asma.ayari@usherbrooke.ca.
}

tals. Thus, our present work describes one approach with the aim of demonstrating the feasibility of this technique for nanocrystal organization, enabling precise control of size as well as of density and spatial position of silicon nanocrystals.

Our nanofabrication process consists of two main steps: (i) electron-beam lithography (EBL) and plasma etching (advanced oxide etching, AOE) to fabricate templates in a silicon nitride thin film, followed by (ii) electrochemical etching of the silicon substrate that produces organized silicon nanocrystals on the surface. A layer of $30 \mathrm{~nm}$ thick silicon nitride $\left(\mathrm{Si}_{3} \mathrm{~N}_{4}\right)$ was deposited using low pressure chemical vapor deposition on a P-doped (100)-oriented silicon substrate with a resistivity of $1-10 \Omega \mathrm{cm}$. In order to prepattern the surface, $\mathrm{Si}_{3} \mathrm{~N}_{4}$ layers were spin coated with $100 \mathrm{~nm}$ of resist, ZEP520 diluted in anisole. ${ }^{13}$ Samples were then annealed at $180{ }^{\circ} \mathrm{C}$ for a few minutes. EBL was performed by means of a LEO1530 scanning electron microscope externally controlled by a computer equipped with lithography software using the Nabity Pattern Generation System. In order to obtain nanosize holes, a single point exposure scheme was used for each point. After exposure, the development of the resist was completed using ortho-xylene. This template is used as a mask for plasma etching. The $\mathrm{Si}_{3} \mathrm{~N}_{4}$ holes were etched through the resist layer using a Surface Technology System advanced oxide etch inductively coupled plasma system in a gas mixture of $\mathrm{CF}_{4} / \mathrm{He} / \mathrm{H}_{2}$. After $\mathrm{Si}_{3} \mathrm{~N}_{4}$ etching, the resist layer was removed using nanostrip followed by an oxygen plasma cleaning to remove any residual organics.

The nanocrystals are then formed at the surface of the silicon substrate through the $\mathrm{Si}_{3} \mathrm{~N}_{4}$ mask by using electrochemical etching. As a result, a monolayer of laterally separated silicon nanocrystals at the surface of the wafer was formed by using a short monopulsed anodization current in the transition regime, ${ }^{9}$ which is a specific regime situated between two other anodization regimes: porosification and electropolishing. ${ }^{14}$ The electrochemical etching was performed in a galvanostatic regime under daylight using an electrochemical cell. The back side of the Si substrate was connected to a small copper anode via an $\mathrm{Al}$ ohmic contact. The electrolyte was prepared using concentrated aqueous hydrofluoric acid (HF 49\%) diluted in ethanol. In this work, we used a 1:7 volume mixture of $\mathrm{HF}$ and ethanol. In order to work in the transition regime, short pulses of etching cur- 


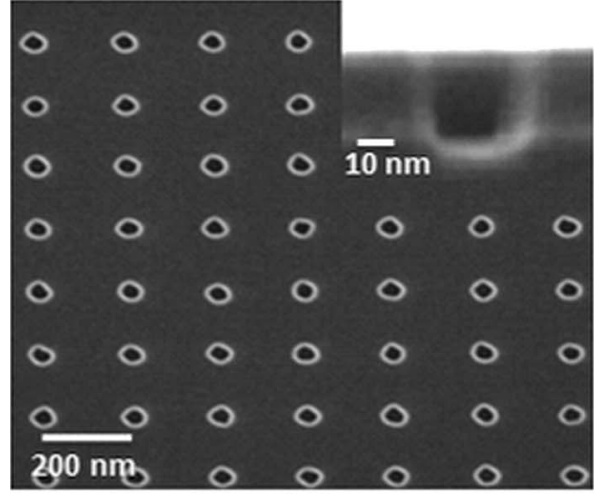

(a)

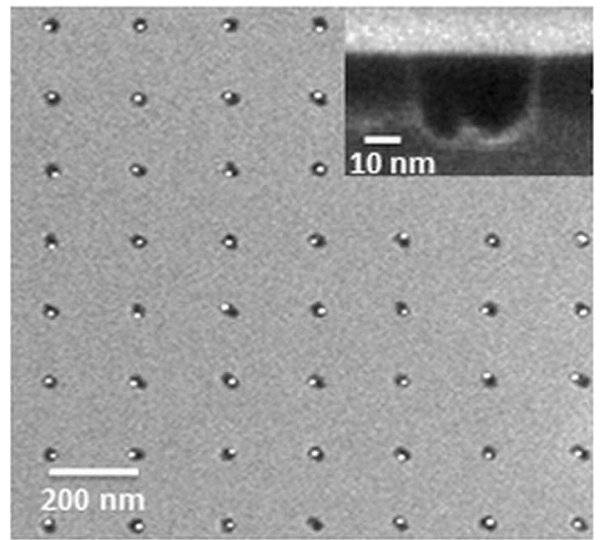

(b)

FIG. 1. (a) $\mathrm{SEM}$ images of nanoholes array in $\mathrm{Si}_{3} \mathrm{~N}_{4}$ layers after plasma etching and resist stripping The inset shows a cross-section image of one hole profile. (b) SEM images of silicon nanocrystals array after electrochemical etching through the silicon nitride nanohole pattern. The inset shows a cross-section image of one hole profile containing one silicon nanocrystal fabricated by the electrochemical etching step.

rents were applied to the cell with different current densities between 4 and $10 \mathrm{~mA} / \mathrm{cm}^{2}$ and for relatively short times between 5 and $10 \mathrm{~s}$ using a programable current source at room temperature. In order to achieve a better characterization of the silicon nanocrystals, $\mathrm{Si}_{3} \mathrm{~N}_{4}$ thin films were etched away with hot phosphoric acid $85 \%\left(\mathrm{H}_{3} \mathrm{PO}_{4}\right)$ at $140{ }^{\circ} \mathrm{C}$. This solution was selected in order to ensure high etching selectivity regarding to silicon. ${ }^{15}$ The surface topography of all samples was investigated by transmission electron microscopy (TEM), scanning electron microscopy (SEM), and atomic force microscopy (AFM) operating in tapping mode.

Figure 1(a) presents an example of SEM image on the surface of nanoholes array obtained in $\mathrm{Si}_{3} \mathrm{~N}_{4}$ layers after plasma etching (AOE) and resist stripping. The inset shows a cross-section image of one hole profile. Mean hole diameter and periodicity were about 30 and $200 \mathrm{~nm}$, respectively. In particular, achieved vertical sidewalls of the nanoholes can be seen on the inset in Fig. 1(a). The surface of the patterned wafer after electrochemical etching is shown in Fig. 1(b). As one can see, ordered array of homogenous silicon nanocrystals localized in prepatterned holes were obtained. A typical cross-section SEM image of the $\mathrm{Si}_{3} \mathrm{~N}_{4}$ hole profile as well as of a single Si nanocrystal formed at the hole bottom after the electrochemical etching can be seen respectively in the insets of Figs. 1(a) and 1(b). By adjusting the anodization parameters (time, HF concentration, and current density), we are

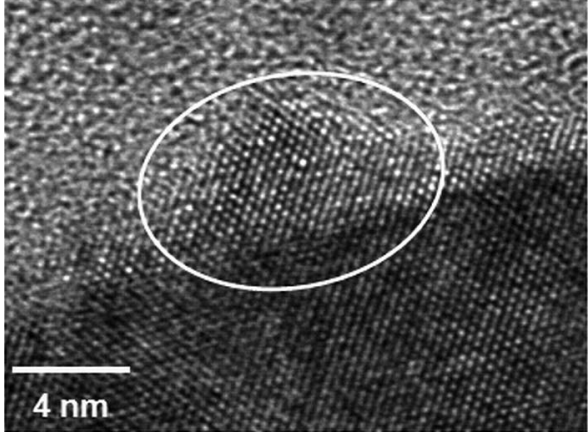

FIG. 2. Cross-sectional high resolution TEM image of a unique silicon nanocrystal obtained by our approach.

able to achieve a very high control of the required silicon nanocrystal density. Particularly, in this example [Fig. 1(b)], anodization parameters were calibrated in order to obtain a single silicon nanocrystal per hole with a very good uniformity across the entire sample. We obtained an ordered array of silicon nanocrystals $\left(200 \times 200 \mu \mathrm{m}^{2}\right)$ with a periodicity of $200 \mathrm{~nm}$. A yield of 99.34 percent was measured on a surface of $2.47 \times 10^{7} \mathrm{~nm}^{2}$ which containing 770 holes. The nanocrystal diameter was $10 \pm 1.2 \mathrm{~nm}$ with a height of $4 \pm 1.6 \mathrm{~nm}$.

Figure 2 shows an example of a cross sectional TEM image of a single silicon nanocrystal realized by this technique. We observe that the silicon nanocrystal (the encircled area) is conical and has the same crystallographic orientation as the $\mathrm{Si}$ substrate. The dimensions of the nanocrystals were about $10 \pm 1.4 \mathrm{~nm}$ in diameter and $4 \pm 1.5 \mathrm{~nm}$ in height.

The profile of a single silicon nanocrystal obtained with an AFM as well as a topographical magnified AFM image of the nanocrystal are illustrated in Fig. 3. Comparing with results obtained by SEM and TEM, lateral diameters measured by AFM are larger than the actual values because of the size of the curvature radius of the AFM probe tip which is similar to the nanocrystal diameter. However, the general localization in the hole, shape and height of the nanocrystals were similar to the observation performed by TEM. The diameter of silicon nanocrystals was measured to be $20 \pm 2.1 \mathrm{~nm}$ with a height of $4 \pm 1.4 \mathrm{~nm}$, localized in holes of about $36 \mathrm{~nm}$ diameters. We thus obtain localized homogeneous silicon nanocrystals. However, to use them in single electron devices, our next work will be devoted to the oxidation of these nanocrystals in order to isolate them electrically from the bulk. Indeed, it was shown that silicon nanocrystals oxida-

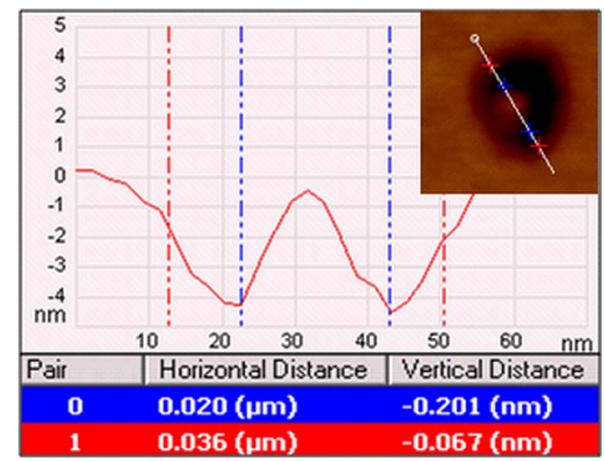

FIG. 3. (Color online) AFM image and a profile of a single silicon nanocrystal inside the $\mathrm{Si}_{3} \mathrm{~N}_{4}$ nanohole. 
tion occurs beneath the nanocrystal as well as on the top and sides in $\mathrm{O}_{2}$ at 850 and $1050{ }^{\circ} \mathrm{C} .{ }^{16,17}$ This is an important point which will be validated in our next studies.

In conclusion, we have demonstrated and validated an approach to obtain two-dimensional ordering of silicon nanocrystals. This approach consists in the localization of silicon nanocrystals via an electrochemical etching in the transition regime on a prepatterned silicon nitride surface. The main advantage of this process is that it allows complete control of the location of the nanocrystals, the distance between them and their crystalline orientation. This achievement can have a major impact on future applications in nanoelectronics devices, in particular for the large-scale fabrication of single electron transistors and nonvolatile memories. This simple low cost approach allows the control of the quantum size effects and single-electron charging in these isolated nanocrystals for nanoelectronics device fabrication. This effort is currently ongoing and more detailed studies on the influence of the parameters of anodization on shape and size of the nanocrystals, their oxidation, and electrical characteristics are under development.

The authors are grateful to Professor A. Jaouad for silicon nitride plasma etching and to all the staff of the center for their technical support for parts of this work.

${ }^{1}$ A. Nakajima, T. Futatsugi, K. Kosemura, T. Fukano, and N. Yokoyama, Appl. Phys. Lett. 71, 353 (1997).

${ }^{2}$ S. Tiwari, F. Rhana, H. Hanafi, A. Harstein, E. F. Crabbé, and K. Chen,
Appl. Phys. Lett. 68, 1377 (1996).

${ }^{3}$ F. Patella, A. Sgarlata, F. Arciprete, S. Nufris, P. D. Szkutnik, E. Placidi, M. Fanfoni, N. Motta, and A. Balzarotti, J. Phys.: Condens. Matter 16, S1503 (2004).

${ }^{4}$ V. Repain, G. Baudot, H. Ellmer, and S. Rousset, Europhys. Lett. 58, 730 (2002).

${ }^{5}$ S. Rousset, V. Repain, G. Baudot, H. Ellmer, Y. Garreau, V. Etgens, J. M. Berroir, B. Croset, M. Sotto, P. Zeppenfeld, J. Ferré, J. P. Jamet, C. Chappert, and J. Lecoeur, Mater. Sci. Eng., B 96, 169 (2002).

${ }^{6}$ A. Karmous, I. Berbezier, and A. Ronda, Phys. Rev. B 73, 075323 (2006).

${ }^{7}$ P. Kitslaar, M. Strassner, I. Sagnes, E. Bourhis, X. Lafosse, C. Ulysse, C. David, R. Jede, L. Bruchhaus, and J. Gierak, Microelectron. Eng. 83, 811 (2006).

${ }^{8}$ A. G. Nassiopoulou, V. V. Gianneta, M. Huffman, M. A. Reading, J. A. Van Den Berg, I. Tsiaoussis, and N. Frangis, Nanotechnology 19, 495605 (2008).

${ }^{9}$ T. Nychyporuk, V. Lysenko, B. Gautier, and D. Barbier, Appl. Phys. Lett. 86, 213107 (2005).

${ }^{10}$ T. Nychyporuk, V. Lysenko, B. Gautier, and D. Barbier, J. Appl. Phys. 100, 104307 (2006)

${ }^{11}$ S. Gardelis, I. Tsiaoussis, N. Frangis, and A. G. Nassiopoulou, Nanotechnology 18, 115705 (2007).

${ }^{12}$ S. Gardelis, A. G. Nassiopoulou, F. Petraki, S. Kennou, I. Tsiaoussis, and N. Frangis, J. Appl. Phys. 103, 103536 (2008).

${ }^{13}$ L. Merhari, K. E. Gonsalves, Y. Hu, W. He, W.-S. Huang, M. Angelopoulos, W. H. Bruenger, C. Dzionk, and M. Torkler, Microelectron. Eng. 63, 391 (2002).

${ }^{14}$ R. L. Smith and S. D. Collins, J. Appl. Phys. 71, R1 (1992).

${ }^{15}$ K. R. Williams and R. S. Muller, J. Microelectromech. Syst. 5, 256 (1996).

${ }^{16}$ K. C. Scheer, R. A. Rao, R. Muralidhar, S. Bagchi, J. Conner, L. Lozano, C. Perez, M. Sadd, and B. E. White, Jr., J. Appl. Phys. 93, 5637 (2003).

${ }^{17}$ F. E. Prins, C. Single, F. Zhou, H. Heidemeyer, D. P. Kern, and E. Plies, Nanotechnology 10, 132 (1999). 